\title{
Treatment of thoracolumbar fracture-dislocation with a trauma limitation strategy: a technical report and 2-year follow-up
}

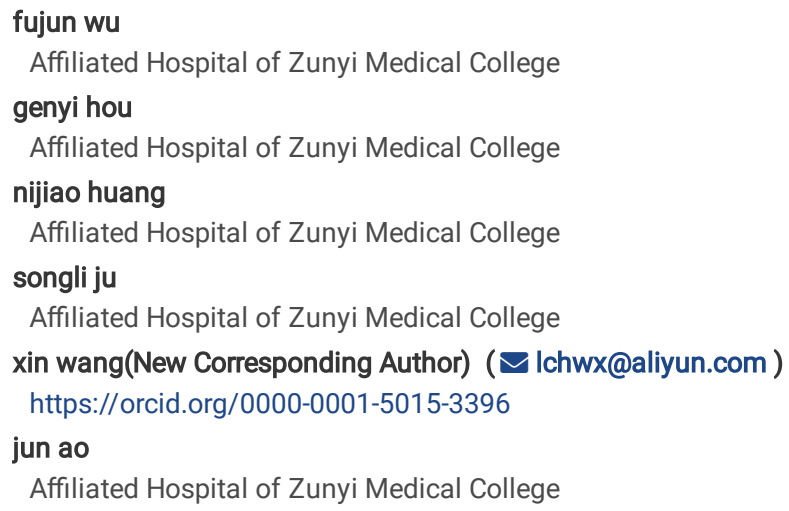




\section{Abstract}

Background: To investigate the curative effect of limited traumatic surgical strategy for thoracolumbar fracture-dislocation.

Methods : Twenty-two patients with thoracolumbar fracture-dislocation who were admitted to our department from May 2013 to January 2016 were 16 males and 6 females, aged 21-53 years. All patients were associated with varying degrees of spinal nerve injury. Twenty-two patients were randomly assigned to the percutaneous minimally invasive group (group A) and the intermuscular approach group (group B). Among them, 11 patients in group A underwent percutaneous minimally invasive pedicle screw fixation combined with small incision for spinal canal reduction. Pressure and interbody fusion: 11 patients in group B underwent transpedicular space fixation with pedicle screw fixation combined with partial decompression and bone graft fusion. The operation time and intraoperative blood loss were recorded in the two groups. Visual analog scale (VAS) was recorded at 3 days and terminal follow-up. The fracture healing and spinal cord invasion rate were evaluated by imaging examination before and after the final follow-up. The neurological recovery was assessed according to the ASIA grading criteria.

Result : There was a statistically significant difference between the two groups in the operation time and intraoperative blood loss (P<0.05). The VAS scores of the lumbar dorsal incision were compared between the two groups at the same time point, and the difference was statistically significant $(P<0.05)$. Imaging examination showed that the fracture healed well and there was no internal fixation fracture. The rate of spinal canal invasion in the fracture and dislocation segments of the two groups was compared at the final follow-up, and the difference was statistically significant. Neurological recovery at the last follow-up (ASIA classification): 6 patients with grade A, 6 patients with grade $B, 7$ patients with grade $C, 2$ patients with grade $D$, and 1 patient with grade $E$, with significant improvement compared with preoperative.

Conclusion: Percutaneous minimally invasive pedicle screw fixation combined with small incision decompression of the spinal canal and interbody fusion can also achieve the effect of posterior open surgery, and the trauma is smaller, which is more conducive to postoperative rehabilitation and reduce surgical complications.

\section{Background}

Thoracolumbar fracture-dislocation is a high-energy injury involving bone and ligament structures that damages the stable spine and often leads to permanent nerve damage ${ }^{[1]}$. Related studies ${ }^{[2]}$ have shown that severe thoracolumbar fracture and dislocation often lead to intervertebral disc and anterior and posterior longitudinal ligament rupture and even pedicle, lamina and articular rupture; the stability of the spine is also severely damaged, and severe cases can endanger a patient's life. Thoracolumbar fracture-dislocation is a three-column injury caused by high-energy complex stress. Treatment should involve early surgical reduction, decompression, bone graft fusion and internal fixation to restore the normal spinal sequence. The stability of the spine should be fully restored, and the recovery of spinal nerve function should be promoted. For these high-energy injuries, the treatment often involves anterior, posterior or anterior-posterior approaches ${ }^{[3-5]}$. Anterior surgery requires a lateral anterior approach for thoracotomy or reconstruction of the anterior middle column spine through the extraperitoneal approach. Due to deeper locations, exposure, more trauma, and greater bleeding, there are few reports on these approaches. The use of the traditional posterior approach is limited due to the extensive exfoliation of the paraspinal muscles and long-term traction during the operation, which destroys the posterior vertebral dynamics and stabilization device, resulting in partial necrosis and fibrosis of the paravertebral muscles. Pain and muscle atrophy are among short- and long-term effects. In recent years, with the development of the minimally invasive concept and the importance of the function of the paravertebral muscles, many spine surgeons have used the paraspinal muscle gap approach to complete the internal fixation of the posterior thoracolumbar spine.

The percutaneous minimally invasive pedicle screw technique, percutaneous pedicle screw fixation and internal fixation for the treatment of lumbar spondylolisthesis, lumbar instability and lumbar vertebrae fractures are the current developing trends in minimally invasive surgery. Compared with traditional open surgery, percutaneous minimally invasive internal fixation or an intramuscular approach induces less trauma and causes less damage to paravertebral muscles, which is more conducive to early recovery and normal function. However, due to certain limitations, percutaneous minimally invasive internal fixation cannot be used for spinal nerve decompression and interbody fusion. Therefore, the optimal treatment strategy for thoracolumbar fracture-dislocation remains controversial. Our department treated 22 cases of thoracolumbar fracture-dislocation with spinal nerve injury from May 2013 to December 2016.

Percutaneous minimally invasive screw fixation and transpedicular space pedicle screw fixation were used. The surgical treatment of combined spinal nerve decompression and interbody fusion for thoracolumbar fracture-dislocation limited the trauma of open surgery to a certain extent and achieved good clinical results. The report is as follows:

\section{Methods}

\section{Clinical Data}

Twenty-two patients with thoracolumbar fracture-dislocation who were admitted to our department from May 2013 to January 2016 were enrolled. There were 16 males and 6 females, aged 21-53 years, with an average age of 40.1 years. The causes of injury were as follows: 10 cases of fall from height, 6 cases of car accident, 4 cases of heavy bruises, and 2 cases of falls. According to the Denis fracture classification, all were fracture-dislocation injuries, with 3 cases of

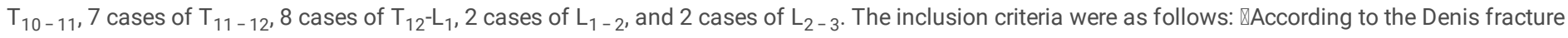
classification, all were fracture-dislocation injuries; खbetween the ages of 20 and 60; $₫$ The patient was admitted to the hospital within 24 hours of the injury; QThe fracture position cannot be higher than T10 or lower than L3, and the fracture-dislocation vertebral body cannot exceed 2 vertebral bodies. Exclusion criteria: $\triangle$ Patients with pathological or severe osteoporotic fractures; $₫$ The patient was severely injured in other areas and could not undergo surgical treatment. 
All patients had fresh fractures with varying degrees of spinal nerve injury. According to the American Spinal Injury Committee (ASIA) grading criteria, 8 patients had grade $A, 6$ patients grade $B, 5$ patients grade $C$, and 3 patients grade $D$ (see Table 1 ).

Table 1

Comparison of nerve damage in the two groups

of patients

\begin{tabular}{|llllll|}
\hline Group & Number of cases & A & B & C & D \\
\hline A & 11 & 4 & 3 & 3 & 1 \\
\hline B & 11 & 4 & 3 & 2 & 2 \\
\hline
\end{tabular}

Preoperative preparation

X-ray, plain CT scan and three-dimensional reconstruction of the injured vertebrae were routinely performed before surgery to determine the fixed segment and decompression range during the operation. Patients were assessed for risk of deep venous thrombosis of the lower extremities before surgery. For patients with prolonged bed rest, serum D-dimer and/or lower extremity venous ultrasound were performed to determine the presence of deep vein thrombosis. Physical prevention and drug prevention were initiated in patients at high risk for thrombosis, and anticoagulant therapy or inferior vena cava filters were initiated in patients with evidence of thrombosis.

Surgical methods

All patients underwent general anesthesia with tracheal intubation in the prone position with the abdomen hanging. In group A, C-arm X-ray was used to project and mark the pedicle of the upper and lower vertebral bodies of the injured vertebrae. After routine sterilization and spreading a towel, the pedicle was located for pedicle screw placement. Once the C-arm X-ray fluoroscopy position was accurate, a $2.0 \mathrm{~cm}$ skin incision was made with the guide needle as the puncture point, the soft tissue was expanded along the guiding installation working sleeve, and the hollow pedicle screw was implanted after the needle was tapped in the sleeve. The posterior median approach centered on the injured vertebrae was approximately $4 \mathrm{~cm}$ long, and the skin, subcutaneous and lumbar fascia were cut open. Subperiosteal dissection of the bilateral erector spinae was performed, revealing spinous processes, lamina, and superior and inferior articular processes; the soft tissue around the dislocated vertebral body was loosened, and the interlocking of the small joints was released. Decompression and contralateral installation of temporary fixation rods was performed to avoid spinal nerve injury due to instability of the spine during decompression. The lateral part of the upper and lower lamina was removed, and the window was opened as close as possible to the inner wall of the pedicle (total laminectomy and decompression were performed if necessary). The dura mater and nerve roots were exposed and protected with brain cotton. If dural rupture is observed during surgery, it should be repaired. During the operation, the spinal cord nerve should be pulled, and the ruptured intervertebral disc tissue should be removed. After decompression, a fixed rod with the appropriate length and curvature should be selected and inserted along the soft tissue channel on both sides. After proper expansion, the vertebral body of the dislocated segment will be pulled and reset. After correction of the C-arm X-ray fracture and dislocation, the ventral side of the dura mater was again examined for broken bone or ruptured intervertebral disc tissue. Allogeneic bone and autologous iliac bone particles were implanted in the injured vertebrae and between the vertebral bodies and between the joints. After rinsing the incision, the drainage tube was placed at the decompression of the window, and the incision was closed. Group B was used to mark the position of the pedicle of the injured vertebrae and the two adjacent vertebral bodies by $\mathrm{C}$-arm X-ray before operation. The middle route was taken, and the towel was routinely disinfected. Next, the skin and subcutaneous tissue were cut, and the lumbar fascia was cut longitudinally on both sides of the spinous process and pulled to the sides to reveal the erector spinae. The potential gap between the longest muscle and multifidus muscle was bluntly separated to the junction of the transverse process and articular process, and the Kirschner wire was positioned. The C-arm X-ray Klein needle was placed in the pedicle position and inserted into the short-tailed pedicle screw. Then, the bilateral erector spinae were removed under the periosteum of the injured vertebra and retracted to reveal the spinous process, lamina, and upper and lower arthroplasty. The joints were resected, and the vertebral interstitial space was opened. The spinal canal decompression and bone graft fusion operation were the same as those in group A. After rinsing the incision, the drainage tube was placed at the decompression of the window, and the incision was closed.

Postoperative treatment

After bed rest, mannitol and hormones were used to reduce the swelling of the operation area. Antibiotics were used to prevent infection of the incision. Analgesia can be administered to relieve postoperative pain. At 48 hours after the operation or drainage volume $<50$ ml, the drainage tube can be removed. During the postoperative period, treatment was supplemented with traditional Chinese medicine, acupuncture, hyperbaric oxygen and nutritional neuropharmacology to promote the recovery of spinal nerve function. One week after surgery, chest and lumbar support was used to perform passive functional training with the lower limbs to prevent complications such as muscle disuse atrophy, deep vein thrombosis and joint stiffness. From March to June, decisions were made to wear a brace, get out of bed, perform functional exercise and use a protective brace according to the recovery of nerve function.

Observation indicators

The operation time, intraoperative blood loss, and kyphosis correction were compared between the two groups. The ASIA grading standard was used to evaluate the recovery of neurological function, and the pain visual analog scale (VAS) was used to evaluate the degree of postoperative incision pain. Fracture healing and the spinal canal invasion rate were assessed by positive lateral radiographs of the spine and CT plain+3D reconstruction.

Statistical methods 
Statistical analysis was performed using SPSS 18.0 statistical software. Analysis of variance was used for comparison between groups. P<0.05 was considered statistically significant.

\section{Results}

All 22 patients underwent surgery successfully. The operation time was 175-310 min in group A, with an average of 254 min; in group B, the operation time was 180-248 min, with an average of $206 \mathrm{~min}$. There was a significant difference in operation time between the two groups $(P<0.05)$. The intraoperative blood loss was 150-350 ml in group A, with an average of $229 \mathrm{ml}$; in group B, it was 200-500 ml, with an average of $343 \mathrm{ml}$. There was a significant difference in intraoperative blood loss between the two groups $(\mathrm{P}<0.05)$. The surgical incision healed well, and no complications, such as infection of the incision and aggravation of nerve injury, occurred. All 22 patients were followed up for 24 to 35 months. There was no significant difference in follow-up time between group $A$ (28.2 months) and group $B(27.6$ months) $(P>0.05)$. The VAS score of the lumbar dorsal incision was obtained 3 days after operation, with $3.18 \pm 0.98$ points in group $A$ and $6.00 \pm 0.89$ points in group $B$. At the final follow-up, group $A$ was $0.82 \pm 0.60$ points and group $B$ was $1.45 \pm 0.52$ points; the difference between the two groups was significant at this time point $(P<0.05)($ Table 2$)$. The preoperative rate of spinal canal invasion in fracture and dislocation was $67.45 \pm 12.29 \%$ in group $A$ and $65.27 \pm 8.67 \%$ in group $B$. The postoperative rate of spinal canal invasion in fracture and dislocation was 6.45 $\pm 2.21 \%$ in group $A$ and $7.64 \pm 2.11 \%$ in group $B$, with significant improvements in postoperative rates compared with preoperative rates in both groups ( $<<$ 0.05). There was no significant difference between the two groups at the same time points $(P>0.05)($ Table 3$)$. Neurological recovery at the last follow-up (ASIA classification) was as follows: 6 patients were grade A, 6 patients grade B, 7 patients grade $C, 2$ patients grade D, and 1 patient grade E. Compared with the preoperative grades, the postoperative grades were significantly improved (Table 4). Imaging examination showed that the fractures healed well, and no complications such as broken nails or broken rods occurred.

Table 2

Comparison of VAS scores of low back pain in two groups of patients $\left(x \pm s, n_{1}\right.$

$$
=11, \mathrm{n}_{2}=11 \text { ) }
$$

\begin{tabular}{|llll|}
\hline Group & Preoperative & 3 days after surgery & At follow-up \\
\hline A & $8.09 \pm 0.70$ & $3.18 \pm 0.98 \#$ & $0.81 \pm 0.60 \#$ \\
\hline B & $8.18 \pm 0.75$ & $6.00 \pm 0.89 *$ & $1.45 \pm 0.52^{*}$ \\
\hline \multicolumn{2}{|l}{ \#P<0.05 compared with preoperative, ${ }^{*} \mathrm{P}<0.05$ compared with preoperative } \\
\hline
\end{tabular}

Table 3

Comparison of the spinal canal invasion rate (\%) in two groups of patients ( $x \pm$ $\left.\mathrm{s}, \mathrm{n}_{1}=11, \mathrm{n}_{2}=11\right)$

\begin{tabular}{|llll|}
\hline Group & Preoperative & 3 days after surgery & At follow-up \\
\hline A & $67.45 \pm 12.29$ & $10.00 \pm 3.41 \#$ & $6.45 \pm 2.21 \#$ \\
\hline B & $65.27 \pm 8.67$ & $10.27 \pm 2.49 *$ & $7.64 \pm 2.11 *$ \\
\hline \#P<0.05 compared with preoperative, $* P<0.05$ compared with preoperative \\
\hline
\end{tabular}

Table 4

Comparison of neurological recovery between the two

groups

\begin{tabular}{|lllllll|}
\hline Group & Number of cases & A & B & C & D & E \\
\hline A & 11 & 3 & 3 & 4 & 1 & 0 \\
\hline B & 11 & 3 & 3 & 3 & 1 & 1 \\
\hline
\end{tabular}

Three days after imaging, imaging examination showed that all patients had good fracture and dislocation correction, and the spine sequence returned to normal. The two groups of patients were followed up regularly, and bone fusion was confirmed by X-ray or CT plain scan $+3 D$ reconstruction. The imaging examination obtained at the last follow-up showed that the internal fixation device was in a good position, and no complications such as loosening or fracture of the internal fixation occurred, as shown in Figs. 1 and 2

\section{Discussion}

The thoracolumbar spine is located at the junction of the thoracolumbar region and is a transition from a relatively fixed thoracic vertebra to a more active lumbar spine. Due to its special anatomy, the thoracolumbar spine is the most common site of spinal fracture. According to the thoracolumbar injury classification and severity score (TLICS), thoracolumbar fracture-dislocation is the most serious type. Because of the relatively small diameter of the thoracolumbar spine, thoracolumbar fracture-dislocation is complicated by spinal nerve injury, and the corresponding symptoms and signs appear ${ }^{[6-8]}$. Thoracolumbar fracture-dislocation has unique pathological features. The posterior pedicle screw internal fixation system is an effective method for the 
treatment of thoracolumbar fractures. Traditional posterior open pedicle screw fixation is clear and easy to operate, and the intraoperative X-ray exposure time is relatively short. Long-segment fixation plus bone graft fusion restores the height of the vertebral body leading edge, resets dislocation, and allows for Cobb angle correction. The exact effect has been achieved in the angle and other areas, and this technique has been widely used in the treatment of thoracolumbar fracture and dislocation. In the posterior median approach, we found that intraoperative exposure requires the removal of the multicleft muscle at the attachment points of the spinous processes, lamina, and articular processes. Extensive exfoliation may damage the posterior medial branch of the lumbar nerve and increase intraoperative blood loss. The amount of exfoliation, in addition to prolonged traction during surgery, may cause ischemic necrosis, fibrosis, muscle denervation, etc., resulting in long-term postoperative low back pain ${ }^{[9-11]}$, low back muscle weakness, etc. Moreover, the problem of prolonged recovery has become increasingly prominent.

The anatomical structure indicates a loose connective tissue gap between the multifidus muscle and longissimus muscle. In 1968, Wiltse et al. [12-14] proposed a pedicle screw method for the treatment of thoracolumbar fractures through the multifidus and longest muscle gap approach, which extensively exfoliated subcutaneous fat tissue from the lumbar back muscle fascia. Postoperative incisions are prone to fat liquefaction and exudation, leading to delayed wound healing and infection complications. The multifissure muscle gap approach has been used to treat thoracolumbar burst fractures, effectively avoiding the complications of postoperative incision nonhealing. Because the patient's intraspinal muscle injury is small, this approach is convenient for early postoperative low back muscle function exercise and rapid recovery, which greatly reduces the incidence of postoperative low back pain. Compared with the posterior open reduction technique, the minimally invasive technique developed in recent years for the treatment of thoracolumbar fractures has the advantages of less trauma, soft tissue protection, and reduced intraoperative blood loss and postoperative complications ${ }^{[15-17]}$. Lehmann et al. ${ }^{[18]}$ found that serum creatine kinase levels were reduced after transcutaneous surgery by measuring creatine kinase activity after soft tissue injury in an animal model. However, for thoracolumbar fractures with displacement, disc injury, combined with spinal nerve injury and other complex spinal trauma, the treatment plan often involves the posterior median approach for open surgical reduction, decompression, and bone graft fusion. Combined with the advantages of percutaneous pedicle screw fixation and the transsphenoidal approach, our department has carried out two different surgical methods, including percutaneous pedicle screw open reduction and fixation combined with small incision window decompression and intervertebral bone graft fusion, as well as the paraspinal muscle gap approach, common pedicle screw open reduction and fixation, local fenestration decompression and bone graft fusion. Both methods can limit the substantial trauma of open surgery to local surgery, which not only achieves the reduction, decompression, fixation and fusion effects of open surgery but also avoids the considerable surgical damage caused by open surgery. Slow down and other shortcomings. Two groups of patients were treated with pedicle screws, which revealed spinous processes, lamina and articular processes. If the small joints were interlocked, they were released and unlocked. We used vertebral interstitial fenestration to detect the presence or absence of rupture of the dura mater in the spinal canal, spinal nerve injury, etc. If dural rupture was found, the cauda equina was paralyzed and carefully returned to the dural sac with a nerve stripper. The posterior margin fracture of the vertebral body was explored and reset by tapping, the spinal cord or cauda equina nerve compression was relieved, and the dura mater was finally repaired to create conditions for the recovery of nerve function. After the thorough decompression of the spinal canal was performed, the dissection was performed again, and the dislocation and recovery of the spine sequence were corrected by the principle of leverage to achieve the same surgical effect as that of conventional open surgery. Thoracic and lumbar vertebrae fracture-dislocation without obvious height loss of the vertebral body can be implanted at the intervertebral and posterior lateral attachments; if the height of the combined vertebral body is lost, the "shell effect" can occur after the internal fixation of the injured vertebrae [19]. When the anterior middle column is insufficient following injury, the internal fixation will bear substantial stress. The internal fixation will fail frequently, resulting in an increase in the Cobb angle and aggravation of kyphosis. Therefore, in the case of preoperative loss of vertebral height, the decompression is reset. After fixation, the upper endplate of the injured vertebrae was restored by the spinal canal, which effectively restored the height of the front edge of the injured vertebrae. Moreover, bone grafting of the injured vertebrae was performed to restore the strength and stability of the anterior middle column of the injured vertebrae to address the "eggshell effect". Some patients suffer from intervertebral disc injury after fracture and dislocation. Because of the lack of blood supply in the intervertebral disc, the ruptured intervertebral disc lacks healing ability. After the reduction, achieve spine stability with simple posterolateral bone graft fusion is difficult. In addition, some joints may be removed during the intraoperative reduction. Therefore, with a sudden increase in the instability of the spine, the ruptured disc tissue and endplate must be completely scraped during the operation. Then, the autologous iliac bone and allogeneic bone particles are implanted in the vertebral body, and the posterolateral bone graft is merged to achieve the anterior middle and posterior columns. Three-column fusion creates conditions for long-term stability and fusion after surgery.

Percutaneous minimally invasive surgery has a relatively long operation time, mainly because the fixed segment is longer. In the early stage, because the percutaneous minimally invasive pedicle screw system has no long-segment surgery to match the connecting rod, only the appropriate length can be used during the operation. Ordinary connecting rods cause certain difficulties in the shaping and placement of rods. Alternatively, percutaneous minimally invasive surgery requires repeated fluoroscopy during the positioning of the $\mathrm{C}$-arm line during the procedure, resulting in a relatively long $\mathrm{X}$-ray exposure time, which poses certain harm to the health of medical personnel and patients. In the paravertebral space approach, the common pedicle screw is inserted into the paravertebral space. The surgical incision is the posterior median incision of the open surgery. The reduction is performed under direct vision, and the difficulty is basically equivalent to that of open surgery. In this study, the limited treatment plan for thoracolumbar fracture-dislocation injury achieved good surgical reduction. In the minimally invasive group, due to the use of minimally invasive pedicle screws, the cost of hospitalization was relatively high. In contrast, due to the use of common pedicle screws, the cost of hospitalization is lower in the transmucosal group than in the minimally invasive group. However, both approaches were superior to traditional open surgery in the effects on paravertebral muscles, intraoperative blood loss, and postoperative pain. The transparaspinal muscle approach is superior to percutaneous minimally invasive surgery in the operation time, intraoperative X-ray exposure, hospitalization, and cost. Percutaneous minimally invasive surgery is superior to the transsphenoidal approach in terms of bleeding volume and postoperative low back pain. There were no significant differences between the two groups in terms of Cobb angle correction and height recovery of the injured vertebral body.

All patients in both groups had postoperative low back pain. After surgery, the dislocation was corrected, and the stability of the spine was achieved, which reduced the patients' lower back pain. In our study, patients wore chest and lumbar support 1 week after surgery to start lower limb function training, and early functional exercise can reduce complications caused by prolonged bed rest (e.g., hypostatic pneumonia, urinary tract infection, deep vein thrombosis of the 
lower extremities, hemorrhoids, osteoporosis, and pathological fractures), improve the body's immunity and improve patients' quality of life. Combined with traditional Chinese medicine, acupuncture, hyperbaric oxygen and nutritional neuropharmacology, some patients had different degrees of spinal nerve function recovery at the last follow-up. Eleven patients with ASIA grade B and C progressed from 1 to 2, respectively, and 2 patients with ASIA Class A had improved function.

\section{Conclusions}

The limited traumatic treatment of thoracolumbar fracture-dislocation has the advantages of less damage, less bleeding, higher safety, a satisfactory reduction effect and decompression of the spinal canal. There was no obvious difference between this treatment and open surgery, which can be used as an effective strategy for the treatment of thoracolumbar fracture-dislocation. Due to the limited number of patients in this department and the lack of large-scale clinical data, the long-term efficacy needs further observation.

\section{Abbreviations}

\section{VAS}

visual analogue scale

ASIA

American Spinal Injury Committee

TLICS

thoracolumbar injury classification and severity score

\section{Declarations}

\section{Ethics Approval and Consent to Participate}

The study is approved by The Ethical Committee of The Affiliated Hospital of Zunyi Medical College, which belongs to the China Association for Ethical Studies. And the Informed Consent (written) was obtained from all patients included in this study.

\section{Consent for Publication}

Informed Consent (written) was obtained from all participants included in this study.

\section{Availability of Data and Material}

The datasets used during the current study are available from the corresponding author on reasonable request.

\section{Competing Interests}

Drs. Fujun Wu, Jun Ao, GenYi Hou, NiJiao Huang, Songli Ju, and Xin Wang declare no competing interests in this study.

\section{Funding}

This research was financially supported by National Natural Science Foundation of China (No.31760266 and 31960209), Scientific Innovation Foundation for Returned Overseas Chinese Scholars of Guizhou Province No.2018-07, 2018 Zunyi "15851 Talent Elite Project", No. National Natural Science Foundation of China 31760266, Doctoral Science Research Startup Funding of Affiliated Hospital of Zunyi Medical University No.2017-01, and Qian Wei Ji Ban Han No.201724.

\section{Authors' Contributions}

FJW and GYH composed and revised the manuscript and figures, respectively. JA performed the surgery and FJW also participated as an assistant. SLJ and $\mathrm{XW}$ collected the clinical materials and completed the table design. XW supervised the project and revised the manuscript. NJH is responsible for the postoperative care. All authors have read and approved the manuscript.

\section{Acknowledgments}

It is highly appreciated for my tutor for his careful modification to this manuscript and thanks for the support from my family.

\section{References}

[1] Xu JG, Zeng BF, Zhou W,et al. “Anterior Z-plate and titanic mesh fixation for acute burst thoracolumbar fracture”. Spine, 2011,36(7):E498-E504.

[2] Nakashima H,Yukawa Y,Ito K,et al. "Combined Posteroanterior Surgery for Osteoporotic Delayed Vertebral Fracture and Neural Deficit in Patients With Parkinson's Disease". Orthopedics, 2009,32(10).

[3] Kaneda K,Taneichi H,Abumi K,et al. "Anterior decompression and stabilization with the kaneda device for thoracolumbar burst fractures associated with neurological deficits". J Bone Joint Surg Am,1997,79(1):69-83. 
[4] Knop CK,Fabian HF,Bastian L,et al. "Late results of thoracolumbar fractures after posterior instrumentation and transpedicular bone grafting". Spine(Phila Pa 1976),2001,26(1):88-99.

[5] Suk SI,Kim JH,Lee SM,et al. "Anterior-posterior surgery versus posterior closing wedge osteotomy in posttraumatic kyphosis with neurologic compromised osteoporotic fracture".Spine(Phila Pa 1976),2003,28(18):2170-2175.

[6] Sudo $\mathrm{H}$,Ito $\mathrm{M}, \mathrm{Kaneda} \mathrm{K}$,et al. "Anterior decompression and strut graft versus posterior decompression and pedicle screw fixation with vertebroplasty for osteoporotic thoracolumbar vertebral collapse with neurologic deficits”. Spine,2013,13(12):1726-1732.

[7] Gelalis ID,Paschos NK,Pakos EE,et al. "Accuracy of pedicle screw placement:a systematic review of prospective in vivo studies comparing free hand,fluoroscopy guidance and navigation techniques”. Eur Spine J,2012,21(2):247-255.

[8] Silbermann J,Riese F,Allam Y,et al. "Computer tomography assessment of pedicle screw placement in lumbar and sacral spine:comparison between freehand and O-arm based navigation techniques". Eur Spine J,2011,20(6):875-881.

[9] Palmer DK,Allen JL,Williams PA,et al. "Multilevel magnetic resonance imaging analysis of multifidus-longissimus cleavage planes in the lumbar spine and potential clinical applications to Wiltse's paraspinal approach”. Spine (Phila Pa 1976),2011,36(16):1263-1267.

[10] Edwards CC,Bridwell KH,Patel A,et al. "Long adult deformity fusions to L5 and the sacrum.A matched cohort analysis".Spine (Phila Pa 1976),2004,29(18):1996-2005.

[11] Hebert JJ,Marcus RL,Koppenhaver SL,et al. “Postoperative rehabilitation following lumbar discectomy with quantification of trunk muscle morphology and function:a case report and review of the literature".J Orthop Sports Phys Ther,2010,40(7):402-412.

[12] Wiltse LL,Spencer CW. “New uses and refinements of the paraspinal approach to the lumbar spine”.Spine (Phila Pa 1976),1988,13(6):696-706.

[13] McCulloch JA,Weiner BK. “Microsurgical techniques for the lumbar spine”. Current Opinion in Orthopedics,2000,11(3):186-191.

[14] Grifka J,Witte H,Faustmann P,et al. "Surgical approach in lumbar intervertebral disk displacement. Topographical principles and characteristics".

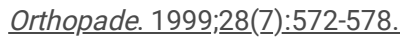

[15]Dickerman RD,Reynolds AS, Tackett J et al.“Percutaneous pedicle screws significantly decrease muscle damage and operative time: surgical technique makes a c EurSpine J,2008,7(10):1398.

[16]Lehmann W,Ushmaev A,Ruecker A et al. “Comparison of open versus percutaneous pedicle screw insertion in a sheep model”.EurSpine J,2008,17(6):857-863.

[17] Lendemans S,Hussmann B,Kauther MD etal. “Minimal-invasive dorsale Stabilisierung der thorakolumbalen Wirbelsäule”. Unfallchirurg,2011,114(2):149159.

[18] Lehmann W,Ushmaev A,Ruecker Aetal. “Comparison of open versus percutaneous pedicle screw insertion in a sheep model”. EurSpine J,2008,17(6):857-863.

[19] Lin D,Guo L,Ding Z,et al. "Modified surgery for acute thoracolumbar fractures:a prospective report". Eur Orthop Traumatol,2011,2(1):33-39.

\section{Figures}
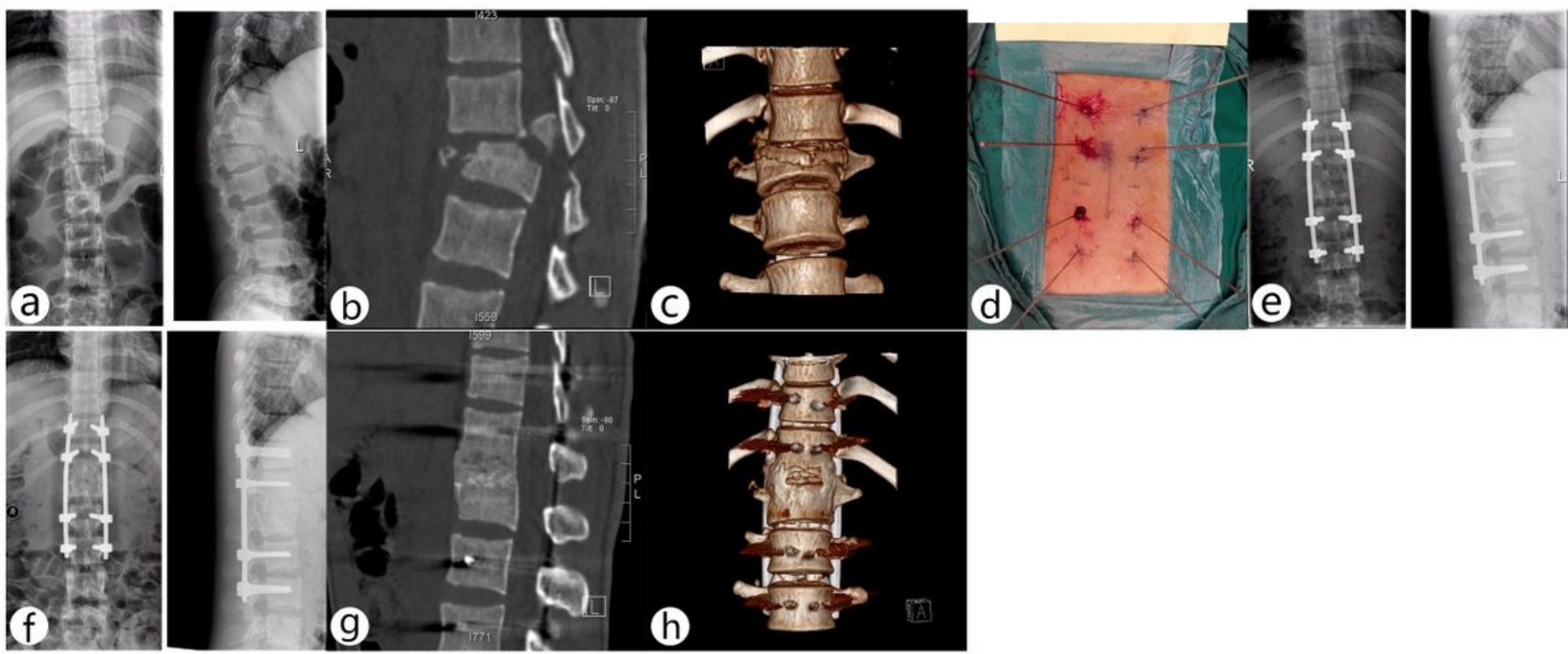

Page $7 / 8$ 


\section{Figure 1}

Male patient, 21 years old, with heavy bruises resulting in T12-L1 vertebral fracture-dislocation (ASIA grade: grade A). a preoperative spine positive lateral X-ray showing T12-L1 vertebral fracture-dislocation; b, c preoperative CT+3D reconstruction showing that the fracture block protruded into the spinal canal; $d$ intraoperative percutaneous puncture Kirschner wire used to locate the pedicle screw entry point; e 3 days after surgery, the X-ray of the lateral position of the spine shows complete correction of the fracture and dislocation, and the spine sequence was normal; $f 1$ year after surgery, the $X$-ray of the lateral position of the spine shows that the height of the injured vertebra was well maintained, and there was no loosening or fracture of the internal fixation; $\mathrm{g}$, $\mathrm{h} 2 \mathrm{years}$ after surgery, CT sagittal reconstruction shows interbody fusion and spinal canal patency, and coronal reconstruction shows the formation of a "bone bridge" around the injured vertebrae.

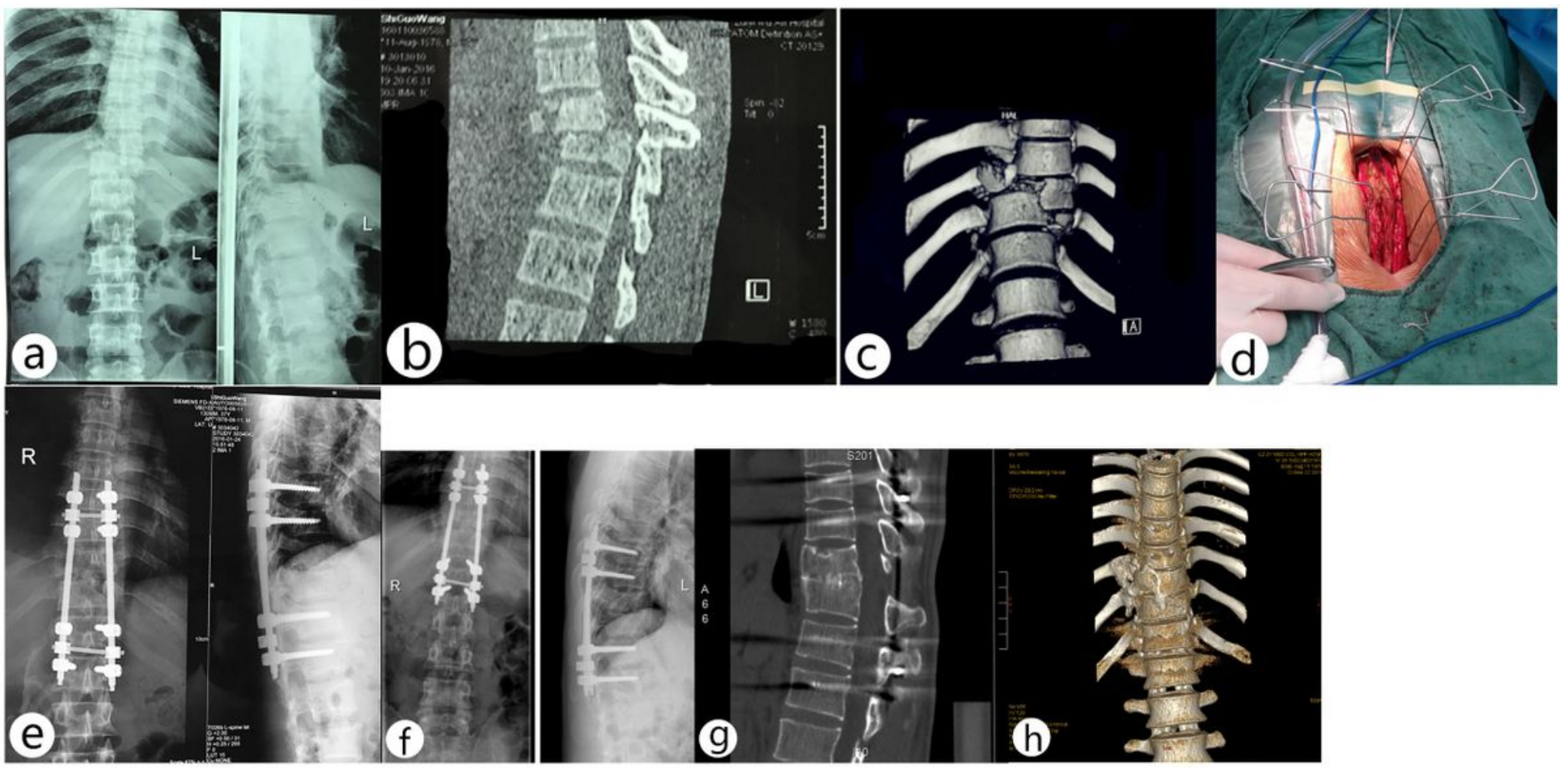

Figure 2

Male patient, 37 years old, with a high fall injury resulting in a T10-11 vertebral fracture-dislocation (ASIA classification: grade B). a preoperative spinal X-ray showing T10-11 vertebral fracture-dislocation; b, c CT +3D reconstruction showing T10 vertebral anterior and left lateral dislocation; $d$ was exposed through the muscle space, and the Kirschner wire was used to locate the vertebral arch. The root insertion point; e 3 days after the operation, the $\mathrm{X}$-ray of the lateral position of the spine shows complete correction of the fracture and dislocation, and the shape of the injured vertebra returned to normal; $f 1$ year after the operation, the X-ray of the lateral position of the spine shows that the height of the injured vertebra remained good; g, h 2 years after surgery, CT sagittal reconstruction shows interbody fusion and spinal canal patency, and coronal reconstruction shows complete morphology of the injured vertebrae. 